\title{
A Cost Sensitiviy Analysis for Carrier Grade Wireless Mesh Networks with Tabu Optimization
}

\author{
David Chieng \\ Malaysian Research Centre \\ BT Innovate and Design \\ Kuala Lumpur, Malaysia \\ david.chieng@bt.com
}

\author{
Dirk v. Hugo \\ Deutsche Telekom Laboratories \\ DTAG \\ Darmstadt, Germany \\ Dirk.von-hugo@telekom.de
}

\author{
Albert Banchs \\ Universidad Carlos III de Madrid \\ Madrid, Spain \\ banchs@it.uc3m.es
}

\begin{abstract}
We present an insight on the sensitivity of total cost (CAPEX+OPEX) towards various key input parameters for CARrier Grade Wireless MEsh Networks (CARMEN). deployment These input parameters span across three main categories namely the network design options, environment conditions and cost. Various boundary conditions are imposed to allow network operator to understand the impacts of parameters' changes with the highest level of uncertainty. A simple Tabu optimization method is adopted to optimize the node density against target data rate and range.
\end{abstract}

Keywords- Wireless Mesh Network, Radio Planning, Cost Analysis

\section{INTRODUCTION}

The input parameters of network / cost model generally come in different ranges of values and boundary conditions. Coupled with different scenario assumptions, this may lead to some degrees of uncertainty on the output. In order to understand the influence of those parameters onto the results, a sensitivity analysis via surface analysis is proposed. Through the this analysis the impact of selected input parameters like coverage, subscriber density, end users' data rate/QoS requirements, network design, equipment, rental costs, etc, on the overall Capital and Operational Expenditure (CAPEX/OPEX) can be better understood. The knowledge of parameter value and result correlations will help network operator to identify the main cost drivers of the deployment scenario, which will then reveal the impact of identified risks on the key financial indicators and will provide transparency between the risk level and profitability [1]. Although cost sensitivity analysis is a typical study [2], to the best of our knowledge no prior study has been understand specifically for (multi-radio) wireless mesh network systems taking into consideration the range of inputs and optimization approach considered.

The rest of the paper is organized as follow: Section II describes the assumptions used. Section III presents the system model. The general parameters and analysis of the results are discussed in section IV. Finally the conclusions and future works are drawn in section $\mathrm{V}$.

\section{ASSUMPTIONS}

In order to derive useful high-level insights concerning the best possible upper-bound capacity performance, the following assumptions have been made:

The research leading to these results has received funding from the EU Framework 7 Programme under grant agreement No. 214994 CARrier grade MEsh Networks (CARMEN). http://www.ict-carmen.eu/)
A two-tier infrastructure mesh network as shown in Figure 1. is considered. The architecture comprises a backhaul tier for providing wireless backhaul connectivity to the infrastructure mesh nodes and an access tier for enabling wireless communication between mesh nodes and client devices. A fraction of these mesh nodes namely CARMEN gateways (CGWs) provide wired backhaul connection to core network. This paper primarily focuses on blanket-like deployment in urban scenario where wireless broadband coverage is typically desired anywhere anytime.

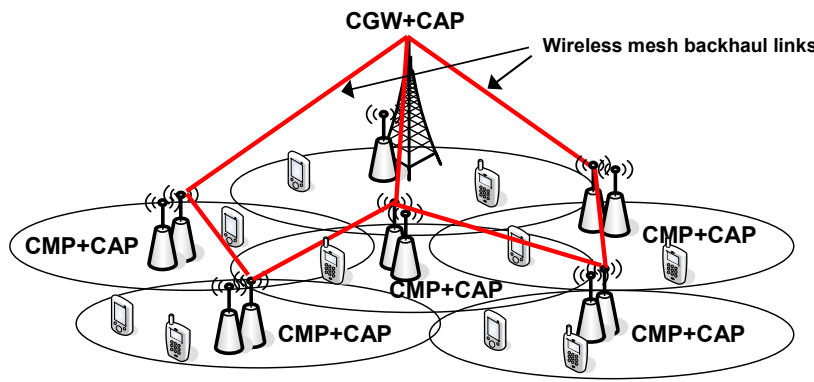

Figure 1. Two-tier network architecture

Each node is equipped with $\mathrm{N}+1$ radio interfaces, in order to create independent backhaul links (non interfering) with its corresponding $\mathrm{N}$ neighbouring nodes and to provide wireless access to its own access domain. It is found that regardless of the formation, clusters of the same size have the same number of radios in total. Various cluster formations based on hexagonal cell layout which is typical in cellular network can be then derived from this architecture (see TABLE I. ).

TABLE I. Cluster Type, CGW-Total Node RATio AND Formation

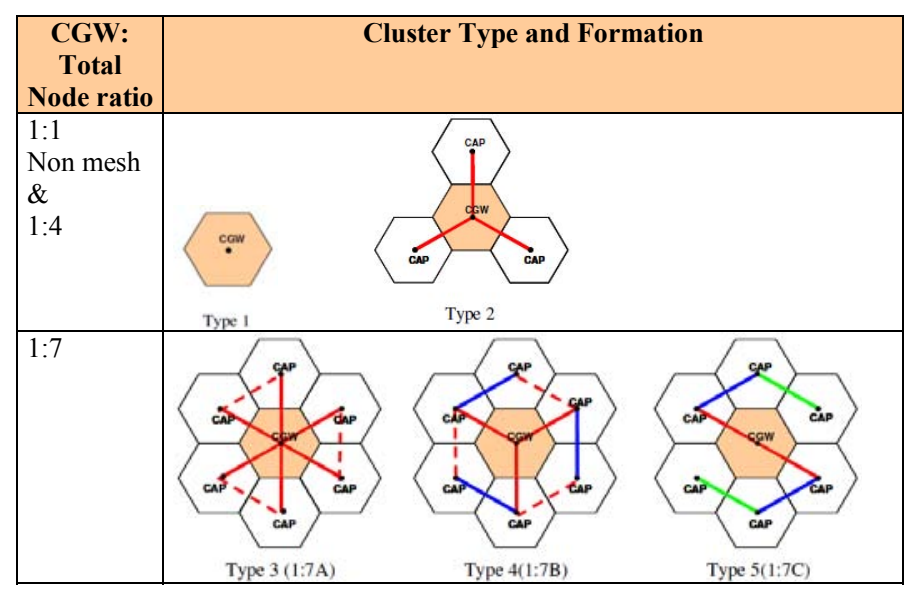




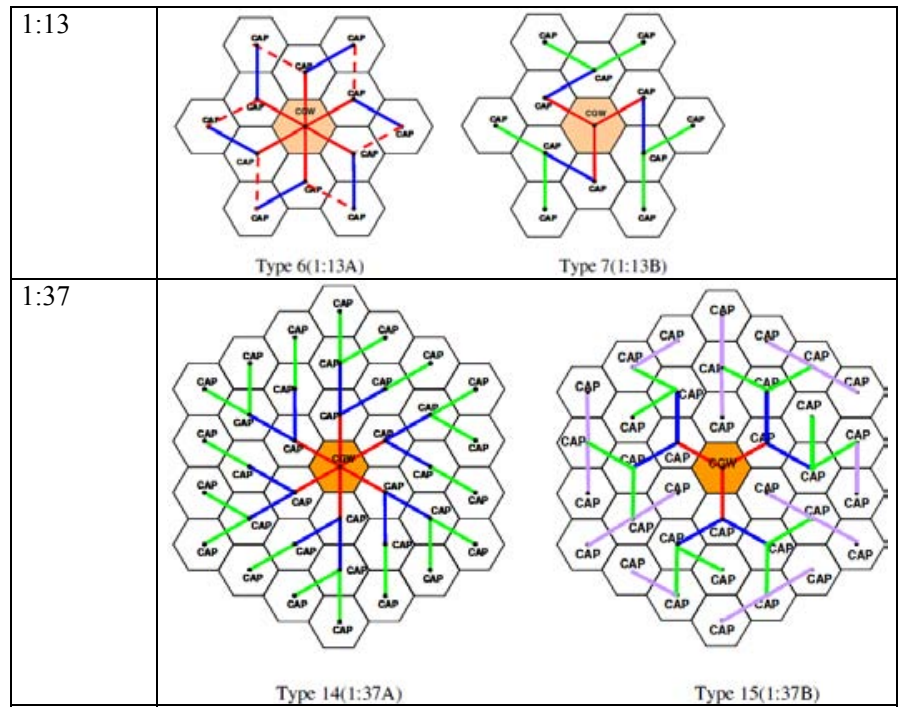

* Note: bold lines denote the primary capacity path while doted lines denote the potential alternative links. Due to space limitation, not all cluster types can be shown here.

Within a cluster, bandwidth is fairly distributed across all CARMEN access points (CAPs) so that nodes with more hops away from a CGW enjoy the same bandwidth as compared to those nodes positioned nearer to the CGW. This can be achieved by applying various well-known techniques described in existing literatures such as traffic admission control at the access point and fair bandwidth scheduling at the CARMEN mesh point (CMPs). It is also assumed that from capacity viewpoint, a mesh network is in fact behaving like a multihop tree when the whole network is operating at maximum load. In other words the alternative paths merely provide resiliency and load balancing but not additional capacity. This assumption is only valid if the network is supported by an effective routing scheme which provides uniform or fair capacity distribution (i.e. load balancing) across all the nodes within a cluster. It is also assumed that $100 \%$ traffic is flowing between users/CAPs and the CGWs.

Subscribers are assumed to be uniformly distributed across the target deployment area and each area may constitute of a number of clusters in repeated pattern. The coverage size per cell is defined by the target data rate to be supported at the cell edge in order to represent the worst case if all subscribers happen to be at the cell edge. Target data rate per user represents the maximum downlink rate that can be enjoyed by an end user which is representative of the connection speed (or headline speed) typically advertised by a network operator. The contention ratio (CR) or overbooking factor is the ratio of the potential maximum demand to the actual bandwidth consumed. A typical CR for "domestic quality" is 50:1 and for "business quality" 20:1. In other words, the lower the CR the higher the mean bandwidth or QoS demand for that service.

Interference arising from co-channel, adjacent channel and foreign devices are assumed to be minimal and can be simply represented as a margin. This assumption is valid in situations where there are sufficient orthogonal channels, and/or the network operates under licensed spectrum [3]. In addition, the effects of interferences can be further minimized through the introduction of smart antenna systems (e.g. beam forming) between stationary mesh nodes. Such assumptions present fundamental requirements for estimating the upper-bound performance limits of wireless mesh networking systems, which are critical for network operators to consider this technology now and its future evolution.

In this study, WiMAX is chosen for analysis. Here the IEEE802.16e Full Usage of Subchannels (FUSC) permutation scheme [4] is adopted to maximize the capacity usage. As for multiplexing scheme, the more popular Time Division Duplex (TDD) mode is selected.

\section{SYSTEM MODEL}

Except for the non-mesh case (Cluster type 1), the coverage area per CAP including $\mathrm{CGW}, A_{\text {mesh }}$ represents a hexagonal cell which is determined by

$$
A_{m e s h}=\frac{\sqrt{3}}{2} D_{m e s h}^{2}
$$

where $D_{\text {mesh }}$ is the distance between two mesh nodes and is given by

$$
D_{m e s h}=2 R \cos 30^{\circ}=R_{m e s h} \sqrt{3}
$$

where $R_{\text {mesh }}$ denotes the cell radius for mesh. For non mesh, the coverage area is given by

$$
A_{11}=\frac{3 \sqrt{3}}{2} R_{11}{ }^{2}
$$

Unlike $R_{\text {mesh }}, R_{\text {l: }}$ is defined by the distance between access point and receiver terminals at the cell edge. Figure 2 . illustrates the relationship between $D_{\text {mesh }}$ and $R_{\text {mesh }}$.

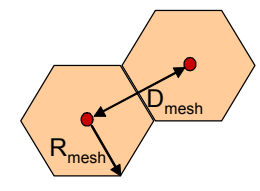

Figure 2. Cell coverage calculation

Based on the fair bandwidth distribution assumption, effective capacity available to user terminals per CAP, $C_{\text {eff }} C A P$ is limited by the effective link capacity at the first hop (counting from CGW), $C_{\text {eff_link }}$ divided by total number of nodes sharing that link or nodes attached to that branch, $N_{\text {branch }}$. Hence,

$$
C_{\text {eff_CAP }}=\frac{C_{\text {eff_link }}}{N_{\text {branch }}}
$$

where $C_{\text {eff_link }}$ also represents the actual capacity of the link used to carry end users' traffic. The statistical average data rate (per direction) per user $\overline{D R}$, is given by

$$
\overline{D R}=\frac{D R_{\text {target }}}{C R}
$$

where $D R_{\text {target }}$ is the target data rate sold by the operator, and $\mathrm{CR}$ is the contention ratio. 
With the knowledge of total number of subscribers per node, the gateway's backhaul $(\mathrm{BH})$ traffics can be derived by aggregating the uplink and downlink traffics from each node. This can be simplified as

$$
B H_{\text {total_traffic }}=\text { TotalNodes } *\left(B H_{D L_{-} \text {traffic }}+B H_{U L_{-} \text {traffic }}\right)
$$

This total backhaul traffic requirement is used to determine the wired backhaul subscription cost that will be described further in section IV.

The general link budget is given by

$$
P L=E I R P-R_{\text {sensitivity }}+G_{r x}-M_{\text {total }}
$$

where $P L$ is path loss $(\mathrm{dB}), E I R P$ is effective isotropic radiated power, $G_{r x}$ is the receiver antenna gain and $M_{\text {total }}$ is the total margin such as shadow, interference, fading, etc.

The pathloss model is based on contributions to the IEEE 802.16 Broadband Wireless Access Working Group by Erceg, et al. [6]. The proposed propagation models cover three terrain categories namely " $\mathrm{A}$ ", " $\mathrm{B}$ ", and " $\mathrm{C}$ " which are representative of urban, suburban and rural environments. These assumptions are suitable for the purpose of this analysis though in practice each environment must be assessed on its' specific characteristics [7].

If assumed full channel (no subchannelization) is allocated to a point-to-point link as in the case of CARMEN, the receiver sensitivity, $R_{\text {sensitivity }}$ (in $\mathrm{dBm}$ ), which is defined as the received signal power needed to operate at a given bit-error-rate (BER) and is given by the following expression [4]:

$R_{\text {sensitivity }}=-114+S N R_{r x}+10 \log \left(\frac{F_{s} * N_{\text {used }} * 10^{-6}}{N_{f f t}}\right)+N F+I L$

where $S N R_{r x}$ is the receiver SNR as listed TABLE II. , $R$ is the repetition factor, $F_{s}$ is the sampling frequency in $\mathrm{Hz}, I L$ is the implementation loss, $N F$ is the receiver noise figure, $N_{\text {used }}$ is the number of used carriers (include data, pilots and dc carriers), $N_{f f t}$ is the number of FFT points. From here, the ideal data rate at the PHY layer can then be represented by:

$$
C_{P H Y_{-} \text {data }}=\frac{N_{\text {data }}}{N_{f f t}} * B W^{*} \text { BitPerSymbol }
$$

TABLE II. RECEIVER SNR ASSUME AWGN WITH REED-SOLOMON CONVOLUTIONAL CODING (RS-CC) AT TARGET BER MEASURED AFTER FEC LESS THAN $10^{-6}[4]$

\begin{tabular}{|c|c|}
\hline MCS & Receiver SNR (dB) \\
\hline BPSK1/2 & 3.0 \\
\hline QPSK1/2 & 6.0 \\
\hline QPSK3/4 & 8.5 \\
\hline 16-QAM1/2 & 11.5 \\
\hline 16-QAM3/4 & 15.0 \\
\hline 64-QAM 2/3 & 19.0 \\
\hline 64-QAM3/4 & 21.0 \\
\hline 64-QAM5/6 & 23.0 \\
\hline
\end{tabular}

where MCS is the target modulation and coding scheme.

\footnotetext{
$1 *$ Not part of main profile but taken from table 532 [4]: Normalized C/N per modulation. For fixed application such as in CARMEN case, this can be easily supported
}

Consequently, the effective downlink capacity can be deduced as

$$
C_{\text {eff_DL_link }}=C_{P H Y_{-} \text {data }} * \operatorname{LinkEff} * T D D_{D L}
$$

where LinkEff is link efficiency representing the capacity loss due to overheads, protocols, etc, $T D D_{D L}$ is the TDD ratio allocated for the downlink. The $C_{\text {eff_DL_link }}$ can be translated to effective capacity per CAP, $C_{\text {eff } D L C A P}$ using equation 4.

With TDD of 3:1 and a nominal channel bandwidth of $20 \mathrm{MHz}$, the resulting DL capacity vs. distance curve across the three geographical environments (terrain types) based on the EIRP limit of $63 \mathrm{dBm}$ is presented in Figure 3. as follows:

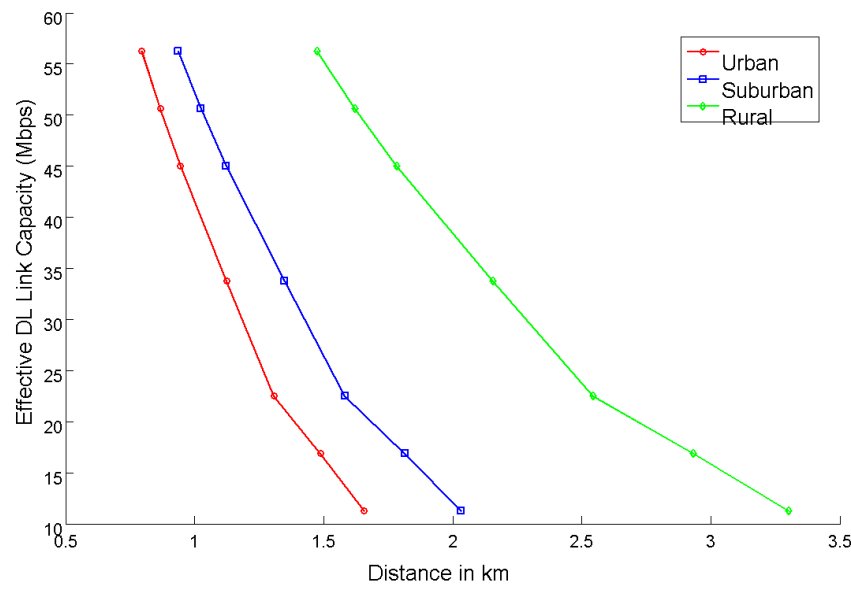

Figure 3. Effective DL link capacity (Mbps) vs. distance $(\mathrm{km})$ across the three environments (or terrain types)

\section{$\underline{\text { Optimization }}$}

The system may operate in either a capacity- or rangelimited case depending on the MCS selected for a link. Here a simplified Tabu optimization method is adopted. In our case, the "tabu" list consists of the minimum node density achievable by a MCS within a cluster type. The goal is to find the minimum node density achievable by each cluster type for a given target input e.g. data rate. The steps are described as follow:

\section{For a cluster type}

Step 1 For a given target input e.g. data rate, $D R_{\text {target, }}$ find the maximum number of subscribers each CAP of cluster type $j$ can support

$$
N_{\text {max }_{-} \text {subs }}^{j}=\frac{C_{\text {eff }}^{j} \bar{D} \bar{L}_{-} C A P}{\overline{D R}}
$$

Step 2 Find the coverage area per node in order to contain this number of subscribers

$$
A_{\text {perDR }}^{j}=\frac{N_{\max _{-} \text {subs }}^{j}}{S D}
$$

where $S D$ is the subscriber density in terms of subscribers per $\mathrm{km}^{2}$.

Step 3: $k \leftarrow 1$. Start iteration with MCS type $k$ and check if $N D_{k}^{j}$ is capacity limited $(C L)$ or range limited $(R L)$

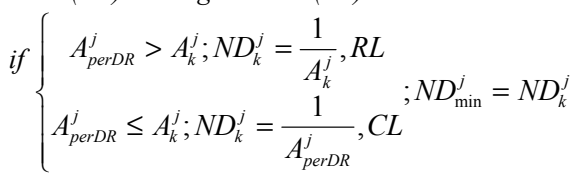

where $A_{k}^{j}$ is the maximum coverage area size achievable by MCS type $k$ 
of cluster type $j$ and $N D_{\min }^{j}$ is the minimum node density for MCS type $k$ of cluster type $j$.

Step $4 k \leftarrow k+1$ while $k<=m$, repeat checking in step 3 and update if a better solution is found.

$$
\text { if }\left(N D_{k+1}^{j}<N D_{k}^{j}\right) ; N D_{\min }^{j}=N D_{k+1}^{j}
$$

where $m=$ max number of MCS as in Table II.

\section{GENERAL PARAMETERS AND RESULTS}

\section{A. General Parameters}

TABLE III. lists the parameters and corresponding values and ranges (if applicable) used for the analyses.

TABLE III. GENERAL PARAMETERS AND TYPICAL RANGES

\begin{tabular}{|c|c|c|c|}
\hline Parameters & Description & Typical Values & Unit \\
\hline \multicolumn{4}{|c|}{ Non technology specific } \\
\hline \multicolumn{4}{|c|}{ General } \\
\hline Cluster type & Type/design of cluster & 1 to 15 & \\
\hline Subscriber density & Number of subscribers per square $\mathrm{km}$ & 100 to 10,000 & Sqkm \\
\hline PathLoss model & Modified SUI & Terrain A B C & \\
\hline Target data rate & Target data rate per subscriber & 0.5 to 10 & Mbps \\
\hline CR & $\begin{array}{l}\text { Contention Ratio - statistiscal mean } \\
\text { access data rate per subscriber }\end{array}$ & $01: 50$ & \\
\hline \multicolumn{4}{|l|}{ Radio } \\
\hline Fc & Centre operating frequency & $2,2.5,3.5,3.7,5$ & $\mathrm{GHz}$ \\
\hline Mesh node Grx & Mesh node's receiver antenna gain & 17 & $\mathrm{dBi}$ \\
\hline UT Grx & User terminal's receiver antenna gain & 6 & $\mathrm{dBi}$ \\
\hline Mesh node NF & Mesh node's Noise Figure & 5 & $\mathrm{~dB}$ \\
\hline Terminal NF & User terminal's Noise Figure & 6 & $\mathrm{~dB}$ \\
\hline IL & Implementation Loss & 5 & $\mathrm{~dB}$ \\
\hline Interference Margin & $\begin{array}{l}\text { Statistiscal average interference that } \\
\text { cause reduction in effective range }\end{array}$ & 3 & $d B$ \\
\hline Htx & Transmit antenna height & 10 & meter \\
\hline Hrx & Receive antenna height & 10 & meter \\
\hline \multicolumn{4}{|c|}{ 至 } \\
\hline CGW Site Cost & $\begin{array}{l}\text { Site acquisition, civil work, installation, } \\
\text { etc }\end{array}$ & 35 & kUSD \\
\hline CAP Site Cost & $\begin{array}{l}\text { Site acquisition, civil work, installation, } \\
\text { etc }\end{array}$ & 5 & kUSD \\
\hline Base radio & Cost per base radio system (wimax) & 15 & kUSD \\
\hline Per radio & Cost per radio interface (wimax) & 7 & kUSD \\
\hline BES Capex & Depending on total backhaul traffic & see reference [5] & kUSD \\
\hline BES Opex & Depending on total backhaul traffic & see reference [5] & kUSD \\
\hline \multicolumn{4}{|l|}{ Technology Specific } \\
\hline \multicolumn{4}{|c|}{ WiMAX (FUSC profile) } \\
\hline Channel bandwidth & Channel bandwidth size & 20 & $\mathrm{MHz}$ \\
\hline TDD ratio & TDD ratio & 3:1 & \\
\hline EIRP limit & depending on channel BW size & 63 & $\mathrm{dBm}$ \\
\hline Link efficiency & $\begin{array}{l}\text { Link efficiency as result of capacity loss } \\
\text { due to overhead (MAC and above) }\end{array}$ & & \\
\hline
\end{tabular}

Since the main interest of CARMEN is to look at carriergrade deployment in the urban areas, the default environment is set as urban with typical urban population of 10,000 per $\mathrm{km}^{2}$. The selected frequency bands vary between 2 to $5 \mathrm{GHz}$ to cover the typical bands used by mobile/fixed wireless broadband services with default at $3.5 \mathrm{GHz}$. Target data rate ranges from $500 \mathrm{kbps}$ to $10 \mathrm{Mbps}$ to represent typical headline rates advertised by current broadband service providers. The contention ratio is fixed at 50:1 to represent typical residential usage pattern. The TDD ratio is set at 3:1 assuming that the subscribers predominantly use downlink intensive applications such as web browsing. As for channel bandwidth, only the $20 \mathrm{MHz}$ channel is studied here due to limited space. There is no standardized maximum limit for WiMAX's Equivalent Isotropically Radiated Power (EIRP) but after studying various online references and recommendations [8], [9], 63dBm is believed to be reasonable for $20 \mathrm{MHz}$ channel. The antenna height is also assumed to be the same for all mesh nodes including the CGW.
Site cost for CGW is assumed to be significantly higher than CAP since CAP nodes are typically much smaller than a base station and are usually mounted on wall, lamp post, phone booth, etc. For wired backhaul service, BT Openreach's Backhaul Extension Service (BES) is adopted. However the cost per km for BES is ignored with the assumption that there is access point presence at the vicinity, which is reasonable for urban area. The detailed pricing structures can be found in [5]. The above pricings are based on the understanding of the authors and should not be interpreted as necessarily reflect BT's official policies or endorsements. There is also no guarantee on the accuracy of cost calculation as pricing and packaging are subject to changes from time to time.

\section{B. Results}

\section{1) Sensitivity Towards Target Average Data Rate}

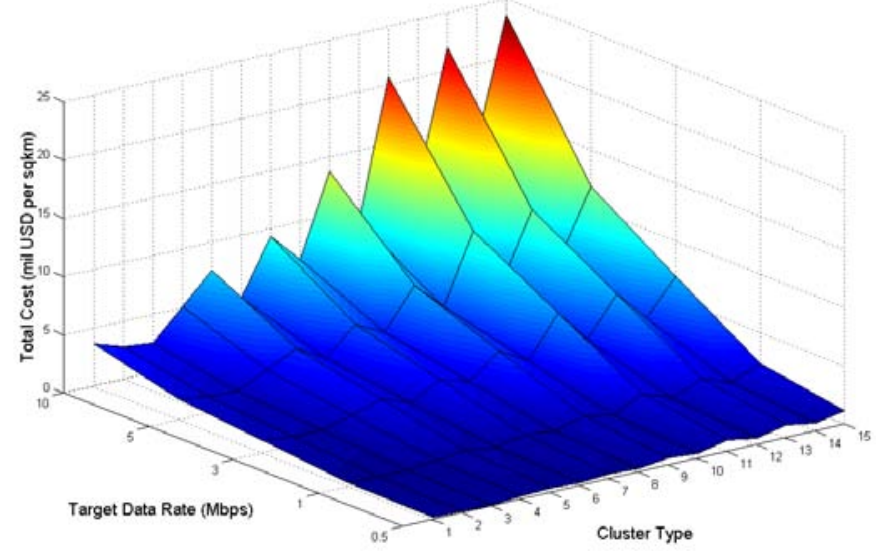

Figure 4. Total Cost vs. Target Data Rate $\left(10,000 \mathrm{subs} / \mathrm{km}^{2}\right)$

The total cost is normalized to unit of a square $\mathrm{km}$ (sqkm) or $\mathrm{km}^{2}$. Figure 4 . shows that across different cluster types, type 2 and 3 are relatively cheaper than non mesh option $(1: 1)$ and this is consistent regardless of target data rates. The most costly option is type 15 with cost per $\mathrm{km}^{2}$ exceeding 1 million USD even though the service provider only wishes to offer merely $0.5 \mathrm{Mbps}$ to all urban subscribers. It is also shown that cluster type $3,6,8,10,12$ and 14 generally perform better than to their peers of the same size types since these clusters have 6 radios at the CGW to share the load.

\section{2) Sensitivity Towards Frequency Band}

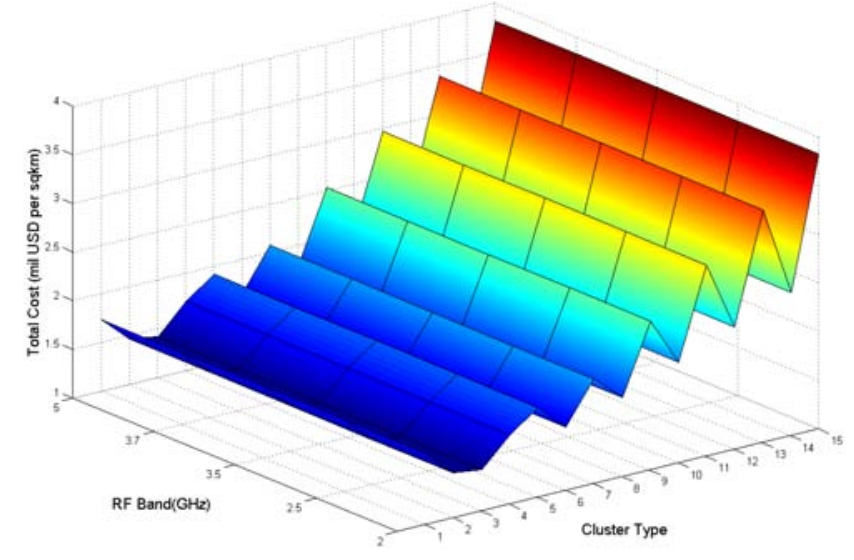

Figure 5. Total Cost vs. RF Band (10,000 subs $\left./ \mathrm{km}^{2}\right)$ 
It can be deduced from Figure 5. that when the subscriber density is high $\left(10,000 \mathrm{subs} / \mathrm{km}^{2}\right)$, the system is operating at severe capacity-limited mode. Due to this no node density reduction and therefore cost can be gained by extending the coverage range using lower RF bands. However as shown in Figure 6. when the subscriber density is low $\left(500 \mathrm{subs} / \mathrm{km}^{2}\right)$ the adoption of lower RF bands generally reduces the cost of most cluster types except type 11, 13 and 15 . When comparing across different cluster types, except for a few larger clusters, mesh general offers better cost benefit over non mesh and this is more significant at higher RF bands.

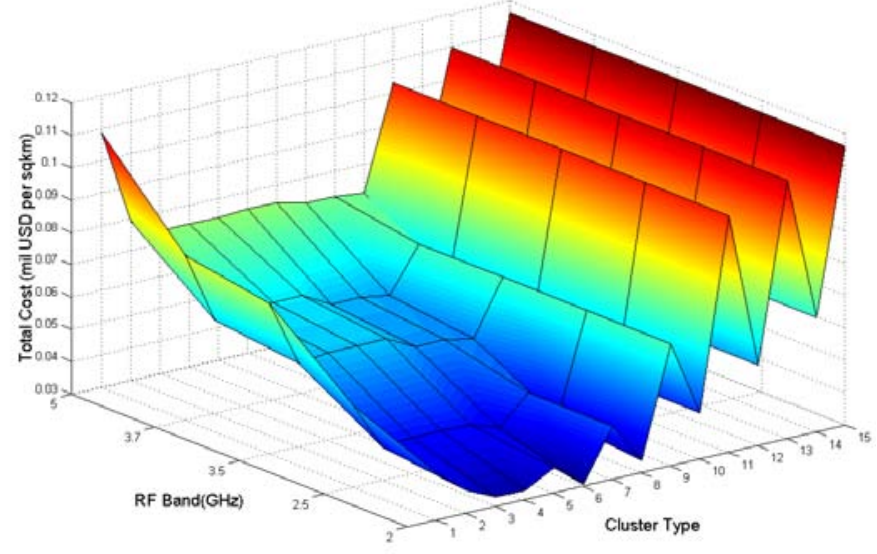

Figure 6. Total Cost vs. RF Band (500 subs $/ \mathrm{km}^{2}$ )

\section{3) Sensitivity Towards Mesh Link Capacity Increase}

The capacity increase represents the usage of advance antenna systems such as MIMO spatial multiplexing. Whether or not to adopt advance antenna systems and when to implement them are some of the common considerations during radio planning.

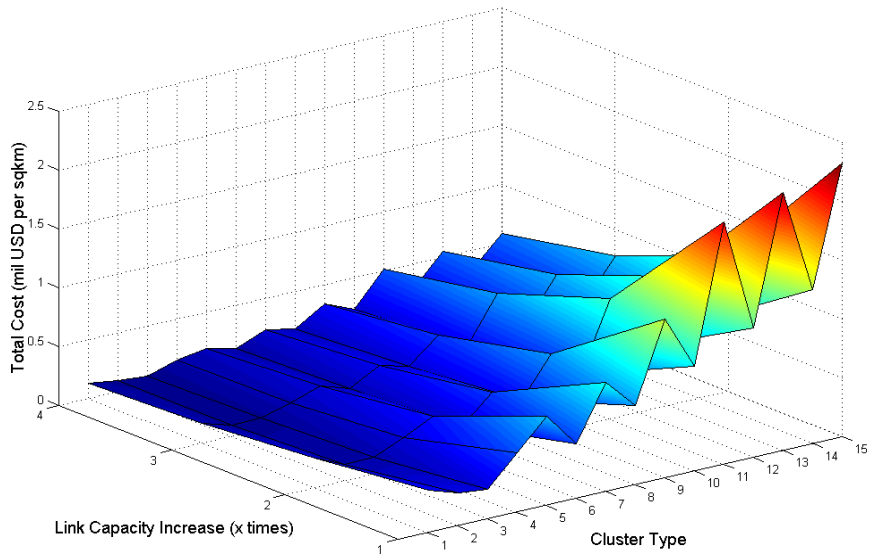

Figure 7. Total Cost vs. Link Capacity Increase $\left(10,000 \mathrm{subs} / \mathrm{km}^{2}\right)$

As shown in Figure 7. , at higher subscriber density, higher link capacity generally helps lower down the total cost i.e. 3fold lower for type 1, 2, 3 and 4 and four-fold for the rest. However when comparing across different cluster types, mesh options do not seem to exhibit much cost benefit compared to non mesh. This is because when operating at capacity limit, any form of capacity increase is beneficial for both mesh and non mesh.
At low subscriber density on the other hand, cluster type 1 to 8 do not benefit much from mesh capacity increase as depicted in Figure 8. This is because at this subscriber density, these clusters mostly operate in range-limited mode. When comparing across cluster types, almost all mesh options are cheaper than non mesh. In fact type 15 becomes the cheapest option with $44 \mathrm{kUSD}$ per $\mathrm{km}^{2}$ when capacity increase factor is $3 \mathrm{x}$ or more.

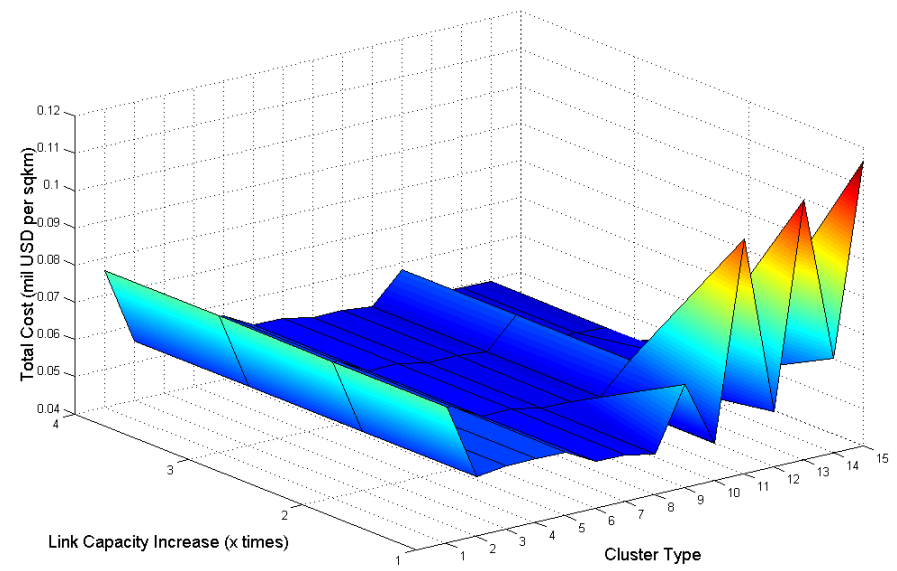

Figure 8. Total Cost vs. Link Capacity Increase (500subs $\left./ \mathrm{km}^{2}\right)$

4) Sensitivity Towards Subscriber Base / Density

In this section, the selected values reflects typical of those ranging from rural to dense urban demographic.

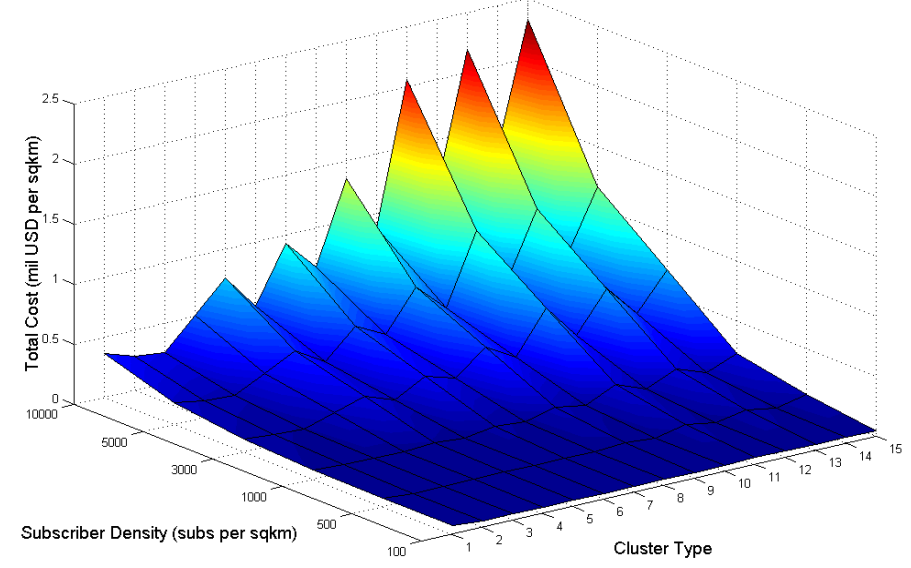

Figure 9. Total Cost vs. Subscriber Density (data rate $=1 \mathrm{Mbps})$

The cost increases with subscriber density in general with small cluster size less sensitive to subscriber density increment. When comparing between mesh and non mesh, most mesh options are only better when subscriber density stays less more than 3000 subs per $\mathrm{km}^{2}$. With higher subscriber density, only type 2 and 3 are cheaper than non mesh. *Note: more discrete values needed.

\section{5) Sensitivity Towards Geographic Environment}

Similar to the case of RF band, it is found that there is virtually no influence from geographical environment on node density since the link is operating at severe capacity-limited condition. At lower subscriber density on the other hand, it is shown in Figure 10. that the total cost is generally lower in 
rural and suburban environments compared to urban but this is only true for smaller cluster types. When comparing between mesh and non mesh, the mesh options are still more attractive in urban-type environment. Only type 2 and type 3 are comparatively better than non mesh across all types of environment.

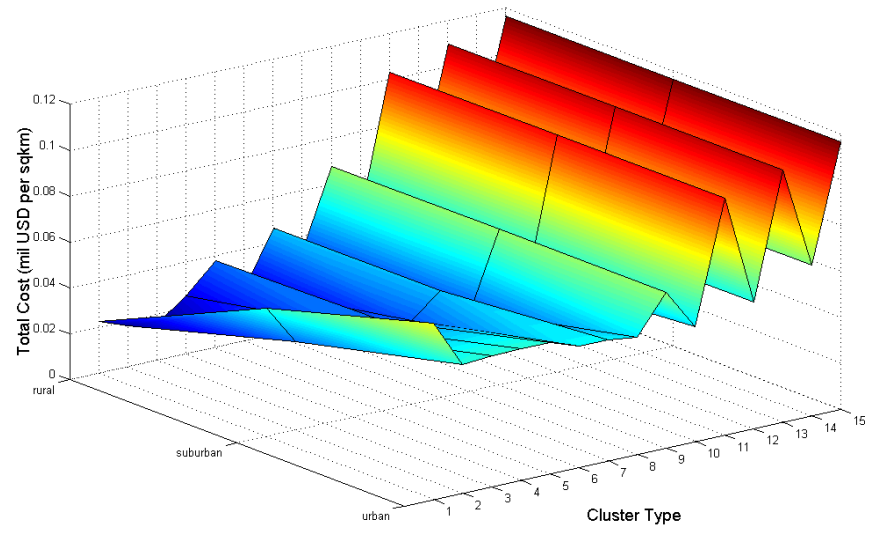

Figure 10. Total Cost vs. Geographical Environment (500 subs $/ \mathrm{km}^{2}$ )

\section{CONCLUSIONS}

In this paper, the sensitivity of total deployment cost (CAPEX+OPEX) towards a number of selected input parameters have been carried out. The input parameters analyzed span across design options, environment conditions and cost. Various boundary conditions have been imposed i.e. high/low data rate, subscriber densities, link capacity, etc., for network operator to understand the impacts of varying those input parameters with the highest level of uncertainty. Due to space limitation however, only a number of key input parameters are investigated. Other parameters such as site cost, radio cost, backhaul subscription cost, etc, are also critical for network operator and can be easily analyzed using the same methodology and model. Nevertheless from this paper, the following observations can be derived:

- The link capacity limit (especially at the first hop between CGW and CAP) remains the primary factor that determines the node density and hence total cost. Whenever the input parameters drive the link to operate in capacity-limited mode, the cost will increase in proportional to the input value.

- The deployment cost is highly sensitive to target data rate and subscriber density that exceeding 1000 subscribers per $\mathrm{km}^{2}$ where the total cost varies in proportional to these parameters, e.g. $1 \mathrm{x}$ increase in target rate resulting in $1 \mathrm{x}$ increase in cost.

- At high subscriber density, RF Band and environment do not have any impact on the total cost. Impacts are only observed at low subscriber density $(\sim 500$ subs $/ \mathrm{km}^{2}$ ).

- The increase of link capacity helps lower down the total cost i.e. $\sim 3$-fold lower for cluster type 1, 2, 3 and 4 and four-fold for the rest.
When comparing across different cluster types, the followings are found:

- $\quad$ Cluster type 2 and 3 are consistently cheaper than non mesh followed by type 4 and 6 which are appealing in some of the scenarios studied.

- The results also show that at lower subscriber density, numerous types of cluster formations can be more attractive than non mesh option. This shows that mesh options in general are better choice during the early phase of deployment and/or when subscriber density is lower than certain threshold. As the subscriber base grows, cluster types can be gradually migrated to other types which can support higher number of subscribers at the target data rate. Such flexibility demonstrates that wireless mesh networks can be easily scaled according to changing subscriber demand without having to install new nodes by simply changing the nodes capability.

\section{ACKNOWLEDGMENT}

The research leading to these results has received funding from the EU Framework 7 Programme under grant agreement No. 214994 CARrier grade MEsh Networks (CARMEN). http://www.ict-carmen.eu/. The views and conclusions contained here are those of the authors and should not be interpreted as necessarily reflect those of BT, DT and U3CM, and also should not be interpreted as necessarily representing the official policies or endorsements, either expressed or implied, of the CARMEN project or the European Commission.

\section{REFERENCES}

[1] Detecon Consulting, "Technical Cost Modelling, A Practical Guide", Opinion Paper, Detecon International GmbH, May 2008.

[2] André Lähr, Kai-Uwe Bletzinger, "Application of Sensitivity Analysis to a Planning Process in Architecture, Engineering and Construction (AEC)", 6th World Congresses of Structural and Multidisciplinary Optimization, Rio de Janeiro, 30 May - 03 June 2005, Brazil.

[3] A. Ting and D. Chieng, "Design and Capacity Performance Analysis of Wireless Mesh Network", International Conference on Mobile Technology, Applications and Systems (Mobility 2008), Ilan, Taiwan, 10 to 12 September 2008.

[4] IEEE P802.16Rev2/D4, Draft Standard for Local and Metropolitan Area Networks, Part 16: Air Interface for Broadband Wireless Access Systems, April 2008.

[5] Backhaul Extension Services (BES), BT Openreach. http://www.openreach.co.uk/orpg/products/ethernet/bes/eoibes.do

[6] IEEE 802.16.3c-01/29r4: Channel Models for Fixed Wireless Applications, Erceg et al., IEEE 802.16 Broadband Wireless Access Working Group, January 2001.

[7] WiMAX Deployment Considerations for Fixed Wireless Access in the $2.5 \mathrm{GHz}$ and $35 \mathrm{GHz}$ Licensed Bands, WiMAX Forum, June, 2005.

[8] Recommendation adopted by the Working Group "Spectrum Engineering" (SE), Electronic Communications Committee (ECC) within the European Conference of Postal and Telecommunications Administrations (CEPT) "GUIDELINES FOR ACCOMMODATION AND ASSIGNMENT OF MULTIPOINT FIXED WIRELESS SYSTEMS IN FREQUENCY BANDS 3.4-3.6 GHz AND 3.6-3.8 GHz", ECC RECOMMENDATION (04)05.

[9] ITU-R, "Characteristics of broadband wireless access systems operating in the land mobile service for use in sharing studies", REPORT ITU-R M.2116, 2007. 